\title{
Ondes de gravité
}

\author{
Jean-Pierre Germain \\ Professeur à l'Université Joseph Fourier de Grenoble
}

C'est un peu une gageure de présenter ce sujet en une dizaine de pages. Il s'agit du problème posé par l'étude des mouvements de fluide en contact avec un gaz, comme par exemple : l'eau de mer en contact avec l'atmosphère, et où les perturbations généralement concentrées vers l'interface, se propagent et se modifient sous l'action de la pesanteur ou gravité. Ce phénomène, a de tous temps intéressé l'homme; le hiéroglyphe égyptien NNN qui se prononce ène et qui représente des vagues schématisées, signifie eau. Au point de vue scientifique, cette branche de l'hydrodynamique remonte à EULER, BERNOULLI et d'AlemBERT, et quoique vieille de plus de deux siècles, elle est toujours d'actualité et fort loin d'être entièrement résolue. C'est dire la difficulté de ce sujet qui du point de vue théorique, pose des problèmes non linéaires à traiter dans un domaine, celui occupé par le fluide, dont une frontière, la surface libre, est elle-même inconnue. C'est l'évolution des idées mises en cuvre, pour trouver des solutions, que nous allons essayer de présenter.

\section{Que d'efforts pour un maigre résultat !!!}

Dans une première étape, il faut mettre le problème en équation. Pour ce faire, on est souvent amené à faire des hypothèses simplificatrices mais raisonnables. Au départ, le liquide est supposé être un liquide parfait (la viscosité est négligée) sans tension superficielle; le fluide est en général mis en mouvement à partir du repos. Les mouvements peuvent être supposés irrotationnels, du moins si les perturbations sont assez régulières. Les obstacles sont généralement imperméables. La surface libre étant inconnue, il est nécessaire d'imposer deux conditions sur celleci. Ces conditions sont : I'imperméabilité, ce qui exclut par exemple l'étude du déferlement, et l'isobarité ou plus généralement une pression imposée, le couplage gazliquide étant faible à l'interface.

Sous cette forme, le problème se ramène à définir par exemple, si nous utilisons le point de vue Eulerien, les 3 composantes de la vitesse, la pression et la dénivellation de la surface libre en fonction des coordonnées spatiales et du temps. Les équations dans la masse traduisent l'incompressibilité du fluide et la loi fondamentale de la Mécanique. Pour achever de déterminer la solution du problème nous devons rajouter les conditions aux limites et les conditions initiales. La conséquence prévisible des difficultés que nous avons signalées précédemment est l'impossibilité d'obtenir la solution explicite du problème, hormis certains cas particuliers.

Il peut-être décourageant de voir, qu'à l'heure actuelle, nous ne connaissons que très peu de solutions exactes du problème outre la solution triviale du repos. Signalons pour les ondes progressives, la solution explicite de GERTSNER [1] datant de 1802, mais avec un rotationnel très particulier. Mais si cette récolte est mince pour une recherche portant sur plus de deux siècles, des développements importants et nombreux ont permis d'approcher la solution des problèmes, et ce sont ces idées que nous allons analyser maintenant.

\section{Au commencement était le repos}

Plaçons-nous dans la situation extrêmement simple d'un problème bi-dimensionnel sur fond horizontal, situation où persistent néanmoins les vraies difficultés du problème (non linéarité, surface libre inconnue a priori).

Ne pouvant résoudre le problème, l'idée qui vient immédiatement à l'esprit est de le linéariser. On peut le faire à la mode des premiers chercheurs, tel AIRY [2] qui aboutit en 1845 à la description de la houle pure ou onde progressive et donna la relation de dispersion qui porte son nom. A cette linéarisation faite à coups de serpe, nous préférons maintenant une présentation plus réfléchie, laissant la porte ouverte à des extensions possibles. Cette méthode, que l'on peut appeler méthode P.P.P. (Petit Paramètre de PoINCARÉ), fut utilisée bien avant que PoINCARÉ ne la systématisât, notamment par STOKES [3] dès 1849. Elle part du fait que, nous connaissons une solution exacte du problème, le repos, et cherche s'il existe des solutions voisines de cette solution particulière. Voisine signifie ici, développable en série entière par rapport à un petit paramètre non fixé a priori, et dont le terme d'ordre zéro décrit le repos. En portant les séries résolutives dans les équations, il faut écrire qu'elles sont vérifiées à chaque ordre. Le système obtenu à l'ordre un est celui de la théorie linéaire, sur laquelle nous allons exposer maintenant un certain nombre d'idées. Mais théoriquement, il faudrait étudier les approximations de tous ordres, et finalement montrer la convergence. Ces étapes ultérieures ne sont pratiquement jamais faites et l'on se contente d'étudier les approximations linéaires, voir celles du second ou troisième ordre.

\section{Théorie linéarisée}

La première et unique forme de mouvement étudiée pendant longtemps fut l'onde progressive ou houle pure de pulsation $\omega$. Si l'on utilise par exemple, la formulation du problème à l'aide du potentiel des vitesses comme inconnue principale, on peut montrer moyennant l'hypothèse complémentaire, que la surface libre reste voisine du 
niveau moyen, ce qui se traduit physiquement par le fait que l'amplitude des mouvements est très faible, qu'il existe pour une pulsation donnée un mode onde progressive qui se propage sans se déformer, et des modes évanescents ou perturbations locales pour lesquelles l'amplitude est une fonction exponentielle de la variable horizontale. Grâce à l'introduction de ce second type de mouvement, généré par la présence d'obstacles et décroissant exponentiellement lorsqu'on s'écarte de ceux-ci, il est possible de résoudre théoriquement tout type de problème sur fond horizontal en présence d'obstacles fixes ou mobiles. Le problème généralement posé est le suivant: une onde incidente donnée attaque un obstacle et engendre deux houles progressives, l'une réfléchie et l'autre transmise, qu'il s'agit de caractériser par des coefficients de réflexion et de transmission, ainsi que des modes évanescents cantonnés au voisinage de l'obstacle et dont la détermination précise est nécessaire à la connaissance fine de l'écoulement dans cette région, et qui permet d'en déduire les pressions, donc les efforts exercés par le fluide sur l'obstacle. Cette prise en compte de ces perturbations est relativement récente. Pour la génération des vagues au moyen d'un bateau, signalons la théorie d'Havelock [4] de 1929. Pour des obstacles fixes, les applications sont encore plus récentes, comme l'étude des barrières verticales immergées qui a été résolue pour la première fois par DEAN en 1945 dans le cas de la profondeur infinie.

En profondeur finie, nous ne possédons pas de solution explicite complète. Le problème se ramène alors à la résolution de systèmes linéaires, d'une infinité d'équations à une infinité d'inconnues. Il ne peut être traité que numériquement, et en général non trivialement, car ces écoulements à potentiel présentent des singularités. Signalons par exemple, le passage d'une houle sur un seuil, étudié en 1960 par TAKANO, élève du professeur J. KRAVTCHENKo, qui a lancé ce genre d'étude à l'Université de Grenoble.

A trois dimensions le problème se complique évidemment, puisque les ondes perturbées peuvent se propager dans une infinité de directions. On a alors à résoudre un problème de diffraction. Là encore, il y a lieu de considérer les modes ondes et les modes perturbations locales. Une simplification existe sur les contours de l'obstacle qui sont verticaux, allant du fond à la surface libre. Dans ce cas, il n'y a pas de modes évanescents, ce qui explique pourquoi les premières études de diffraction se soient faites sur ce type d'obstacles (cas d'une digue semi-infinie attaquée par une houle pure en incidence quelconque). Une difficulté supplémentaire apparaît. Si le milieu est infini, l'amplitude d'une onde peut tendre vers zéro lorsqu'on s'éloigne infiniment de l'obstacle tout en conservant une énergie finie, ce qui permet des entrées "clandestines " d'énergie dans le système. Pour éviter cela et préciser de manière unique la solution du problème, il faut imposer une condition supplémentaire dite de radiation. Ces travaux se rattachent immédiatement à SOMMERFELD.

Pour étudier la propagation des ondes sur un fond lentement variable ou réfraction, il faut toujours pour ce problème linéaire utiliser d'autres méthodes, notamment la méthode W.K.B. qui décrit le phénomène par phase et amplitude. La conduite des calculs est très analogue à celle de l'optique géométrique et est pour cela appelée théorie des rayons.

Elle justifie la méthode autrefois employée par les ingénieurs qui étudiaient les plans de vagues au voisinage de la côte, et qui supposaient qu'une onde "s'adapte " à la profondeur. Les travaux correspondants sont ceux de MEI et LiU [6] de 1977 et de BerKHOFF [7] de 1972.

Ces différentes techniques, permettent à l'heure actuelle, une approche théorique assez complète de la propagation des ondes de gravité dans le domaine linéaire, c'est-à-dire pour de faibles amplitudes relatives.

\section{Toujours plus haut}

L'étude des ordres supérieurs s'impose, dès que l'on a résolu le problème linéaire, et ceci pour plusieurs raisons. Tout d'abord souvent l'expérience confirme les résultats obtenus, mais aussi ces résultats permettent de mettre en évidence des phénomènes nouveaux. Le pionnier en la matière fut StOKES [3] qui ne s'intéressa qu'aux phénomènes les plus simples, c'est-à-dire aux ondes progressives sur fond horizontal. La théorie de STOKES au troisième ordre est couramment utilisée. Mathématiquement, ces théories du second, troisième, ... ne sont guère plus difficiles à résoudre que la théorie linéarisée ; en effet, au lieu d'obtenir un système linéaire homogène, on obtient un système linéaire dont seul le second membre, alors non nul, va en se compliquant avec l'ordre, et donc il faut trouver une solution particulière. Sans entrer dans les détails, notons que, si à l'ordre un dans le domaine nous avons deux ondes de pulsations respectives $\omega_{1}$ et $\omega_{2}$, il apparaît au second ordre des nouvelles ondes dites d'interaction de pulsation $\omega_{1} \pm \omega_{2}$. Dans le cas d'une seule onde, ceci conduit à la mise en évidence du premier harmonique de l'onde. A l'ordre trois suivant le même schéma, apparaissent les interactions du phénomène linéaire avec celui du second ordre, et en particulier les harmoniques d'ordre deux et ainsi de suite. Une application importante consiste à étudier le spectre et à voir quels sont les harmoniques significatifs, ce qui indique jusqu'à quel ordre il faut pousser la théorie pour une description correcte. Au-delà du troisième ordre le problème est désespéré.

L'équipe Grenobloise du Professeur J. Kravtchenko, a beaucoup œuvré dans le domaine de l'interaction dans le cas bidimensionnel. Citons les travaux de FONTANET sur la génération des ondes au second ordre et ceux de DAUBERT sur le troisième ordre en général. Parallèlement sur le problème de la génération du second ordre, nous ne saurions passer sous silence, les études de BIESEL du Laboratoire Dauphinois d'Hydraulique, puis de SOGREAH. C'est à O.M. PHILIPPS que I'on doit les études tridimensionnelles des interactions, qui sont si importantes à l'heure actuelle pour les études de stabilité des ondes.

\section{Les solitons attaquent}

Vers 1840, Russell [8] ingénieur chargé de la surveillance des canaux en Ecosse, pouvait du haut de son cheval, suivre la propagation d'une onde bizarre, constituée d'une seule intumescence, se propageant sur plusieurs miles sans pratiquement se déformer. Cette onde était générée, soit par l'arrêt brusque d'une péniche, soit par la fermeture d'une écluse.

Le concept d'une onde progressive, dont la longueur d'onde était infinie, ou onde solitaire était né. L'existence de cette onde fut d'abord niée par AIRY, et même dans un premier temps par STOKES. En effet, dans la théorie P.P.P. 


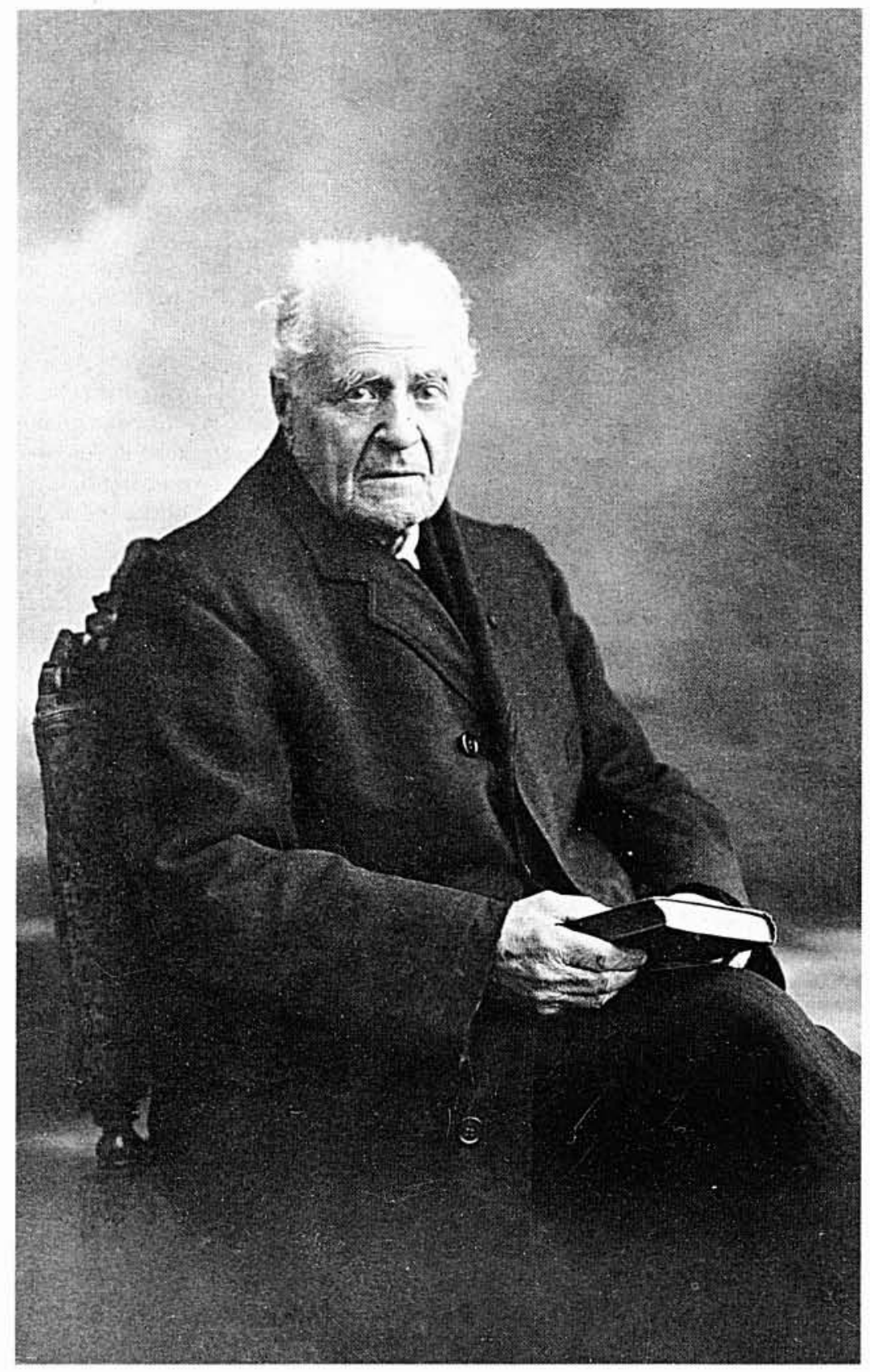

Joseph BOUSSINESQ 1842-1929 
pour les ondes progressives, le petit paramètre est de l'ordre de grandeur de la cambrure, rapport de l'amplitude à la longueur d'onde. Pour une onde solitaire, ce premier à la débusquer fut BoussinesQ [9] en 1871, qui prit en compte que pour une onde de ce type il y a des effets d'échelle différents pour les variables horizontales et verticales.

L'onde solitaire apparaît comme solution d'une équation différentielle non linéaire, mais facilement intégrable, et donnant un profil en $1 / \mathrm{ch}^{2}$ (fig. 1).

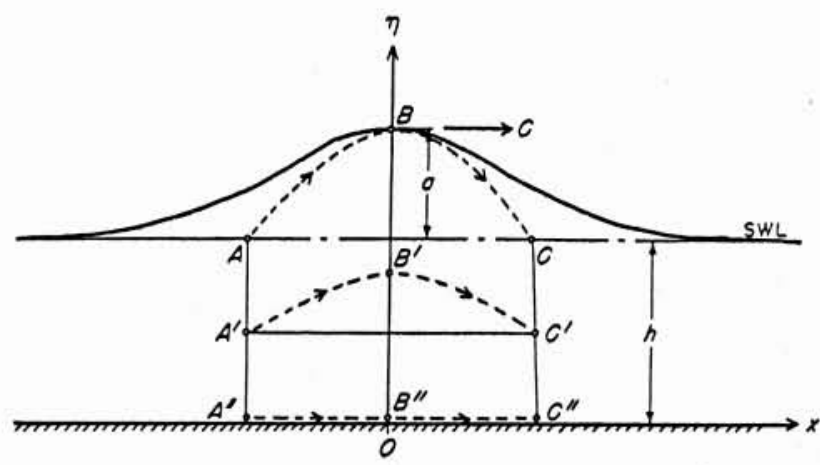

1. Profil de l'onde solitaire et trajectoire des particules Boussinesq :

$$
\eta(x, t)=a \operatorname{sech}^{2}\left(\sqrt{\frac{3 a}{4 h^{3}}}(x-C t)\right)
$$

célérité :

$$
C=\sqrt{g h}\left[1+\frac{a}{h}\right]^{1 / 2} .
$$

Ces résultats furent améliorés quelques années plus tard par KorTEWEG, et Devries [10] en 1805 qui, par le mouvement d'ondes bidimensionnelles se propageant dans un seul sens, obtiennent l'équation dite depuis $K d V$ : équation aux dérivées partielles non linéaires indiquant l'équilibre entre les termes non-linéaires et les termes dispersifs. Cette équation admet des solutions du type ondes progressives. Ce sont des ondes périodiques décrites au moyen de la fonction elliptique cosinus amplitude ou $\mathrm{CN}$. Elles sont appelées houles cnoïdales, et elles admettent comme cas limite l'onde solitaire. A la suite de cela, l'intérêt pour l'équation $K d V$, que l'on ne savait pas résoudre dans le cas général, est retombé.

C'est l'avènement du calcul numérique qui a relancé les études. FERMi, Ulam, et PASTA étudiant numériquement l'évolution dans le temps et l'espace d'une perturbation initialement limitée et vérifiant l'équation de $K d V$, observèrent les résultats surprenants suivants : l'évolution tend à mettre en évidence tout d'abord un train d'ondes solitaire en nombre fini, rangées par ordre d'amplitude décroissant, ce qui est normal car la célérité d'une onde solitaire est en fonction croissante de son amplitude et toujours supérieure à la célérité critique $g h$; ce train peut être vide, puis se propageant avec une célérité inférieure à la vitesse critique un train d'ondes dispersif dont l'amplitude décroît en $t^{-1 / 3}$. De plus, si on fait interagir des ondes solitaires se propageant dans le même sens, le phénomène est assez complexe, mais au bout d'un temps assez long chaque onde réapparaît. L'interaction finale ne se manifeste que par un déphasage.

Ces ondes se comportent comme des particules, et c'est pour cette raison qu'elles sont dorénavant cataloguées comme « solitons ». L'explication de ce phénomène ne fut donné que lorsque KRUSKAL, MIURA, GARDNER, montrèrent comment résoudre l'équation complète de $K d V$. Le processus est assez complexe. Grossièrement, la solution inconnue est considérée comme un potentiel dont on recherche les valeurs propres et les fonctions propres. On peut, à partir du fait que ce potentiel est solution de $K d V$ montrer que le spectre est indépendant du temps ainsi que le comportement final des fonctions propres. On peut donc les calculer à partir de la seule condition initiale qui est donnée. Ces renseignements suffisent à reconstituer la solution à chaque instant, grâce à l'équation de GELFAND. Levitan. Cette méthode est appelée: méthode de la diffraction inverse ou "inverse scattering method". Depuis, ces méthodes ont été couramment employées surtout d'ailleurs dans les laboratoires de recherche.

Comme toujours, dans une première étape, on s'est intéressé aux ondes en l'absence d'obstacles. La présence de ceux-ci entraîne une difficulté majeure. Pour les ondes «longues» au moins en première approximation, la composante horizontale du champ des vitesses est toujours uniforme sur une verticale, donc en général incompatible avec la présence d'obstacles. L'équipe « Ondes de gravité » du laboratoire LEGI de Grenoble a pu pallier cet inconvénient en introduisant dans cette théorie, les modes évanescents. Le résultat intéressant est le suivant : le soliton franchit les obstacles sur fond horizontal sans se casser. $\mathrm{Si}$, par contre au passage de l'obstacle, le fond admet une surélévation, le soliton se casse en au moins deux solitons, et il y a un soliton réfléchi. Si au contraire, le fond est abaissé, il n'y a qu'un soliton transmis et pas réfléchi.

Ces méthodes de diffraction inverse ont été étendues à d'autres configurations. Problèmes à symétrie cylindrique, grâce à l'équation de $K d V$ cylindrique. Problème presque bidimensionnel avec l'équation de KADOMTSEVPetviachVili. Soliton enveloppe et équation de SCHRÖDINGER cubique...

Il existe des cas, où l'on a obtenu l'équation non linéaire, mais où l'on ne connaît pas la méthode $d$ 'inverse scattering correspondante, comme par exemple : l'équation d'OSTRUVSKI en milieu tournant, et même des cas où l'on n'a pas l'équivalent de cette équation.

Pour l'aspect numérique, on préfère utiliser une modification qui prend en compte partiellement une variation verticale de la composante horizontale du champ des vitesses. C'est l'équation de SERrE.

La question que l'on doit maintenant se poser est le lien qui doit exister entre les ondes décrites par la méthode P.P.P. ou ondes courtes, et celles décrites par les équations de BoussinesQ, $K d V, \ldots$ à effet d'échelle que l'on regroupe sous le vocable d'ondes longues.

Dans le cas des ondes progressives, on peut dire que les ondes courtes ont une célérité qui peut prendre n'importe quelle valeur inférieure à la célérité critique. Par contre, les ondes longues ont une célérité voisine de la célérité critique et qui est toujours supérieure à la célérité critique pour les ondes solitaires.

Pour résumer, le phénomène physique admet là deux représentations mathématiques possibles dont le domaine de validité n'est pas identique, même si elles possèdent une région commune.

\section{Solutions exactes}

Elles sont peu nombreuses, et concernent uniquement le cas des ondes progressives. Pour les ondes courtes, LevI- 
Civira a obtenu un théorème d'existence pour les houles interactionnelles en profondeur infinie. Son élève STRUIK en 1925 a étendu ceux-ci au cas de la profondeur finie. Gouyon, élève de KRAvTCHENKo, a pu prendre en compte le cas d'un rotationnel faible. Pour les ondes longues, le cas de l'onde solitaire a été résolu en 1947 par LAVRENTIEU au moyen de représentations quasi conformes, puis par analyse fonctionnelle par FRIEDRICHS et HYERS. LITTMANI a fait de même pour les houles cnoïdales.

J'ai pu montrer que les développements en série des houles longues étaient toujours divergents, mais présentaient le caractère de séries asymptotiques, donc étaient utilisables pour les calculs. Le plus beau mais, aussi le plus " inutile " des théorèmes de cette série, est dû à KrassovSKI. Le plus beau, car il a su montrer sous la seule hypothèse que l'angle des tangentes à la surface libre avec I'horizontale est inférieur à $\pi / 6$ qu'il existait des ondes progressives sans faire appel à l'aspect court ou long. Le plus inutile car c'est un pur théorème d'analyse fonctionnelle, sans possibilité de construire la solution.

\section{Quo non-ascendam}

Nous venons de donner un panorama assez complet de ce que nous pouvons faire en théorie des ondes de gravité, mais avec l'hypothèse de mouvements relativement faibles.

D'autres domaines existent. Les ondes de grandes amplitudes relatives. Jusqu'où l'amplitude d'une onde peut-elle croître avant de se détruire par déferlement?

Il est admis que l'onde limite présente un point anguleux à la surface libre, tout apport supplémentaire d'énergie provoquant la destruction.

Comme toujours le point a été étudié pour commencer, sur le cas des ondes progressives.

STOKES tout d'abord, s'est intéressé au voisinage du point anguleux en écoulement irrotationnel permanent, mettant en évidence un angle de $\pi / 6$ entre la tangente au profil et l'horizontale. Des études systématiques furent conduites par MiCHELL. puis plus systématiquement par YAMADA pour les houles progressives périodiques limitées, obtenant des valeurs pour l'amplitude maximale relative de l'ordre de 0,7 .

Plus récemment, notamment sous l'impulsion de Longuet-Higgins et de Cokelet, la déformation des houles juste avant le déferlement a pu être étudiée.

Malheureusement, sur le déferlement lui-même, nous n'avons pas encore d'ouvertures définitives. D'autres phénomènes ont été négligés dans cet exposé et qui auraient mérité d'y être développés.

Un cheminement analogue des idées s'est manifesté, dans le domaine de la résolution numérique des équations, qui est absolument nécessaire dans les applications, en océanographie physique et océanographie côtière.

Puisque nous essayons de mettre en évidence l'apport grenoblois à nos problèmes, signalons pour les phénomènes de marées à l'échelle d'une mer, voire même de l'océan, l'équipe, sous la direction de Ch. Leprovost et pour l'océanographie côtière et aménagements littoraux par la SOGREAH et le Laboratoire d'Hydraulique de France.

\section{Grenoble sur mer}

Il est bien évident que, toutes les théories que nous venons d'exposer ne présentent d'intérêt pratique que si elles sont vérifiées expérimentalement. La vérification in situ, outre le fait qu'elle nécessite des moyens énormes en hommes et matériels, est en général si complexe qu'il est difficile de faire des tests précis. Par contre l'expérience en laboratoire est beaucoup plus riche en possibilités et c'est ce qui explique le renom grenoblois en la matière, bien que la ville ne soit pas précisément située en bord de mer.

Depuis longtemps on y a travaillé, soit sur les modèles réduits du Laboratoire Dauphinois d'Hydraulique, puis de SOGREAH, ainsi que sur les canaux à houles construits à l'Université par notre regretté collègue MARCOU.

A l'heure actuelle, l'environnement grenoblois dispose dans ce domaine de trois installations remarquables parmi de nombreuses autres. Tout d'abord, un canal à houle tout en glace de $36 \mathrm{~m}$ de long et $0,5 \mathrm{~m}$ de large où sont réalisées les expériences fines bidimensionnelles.

Pour les problèmes tridimensionnels, il existe depuis peu, un bassin de $40 \mathrm{~m} \times 40 \mathrm{~m} \times 1 \mathrm{~m}$ muni d'un batteur serpent, c'est-à-dire formé de 76 volets situés sur un côté et qui peuvent être pilotés indépendamment.

Enfin, unique au monde, la plaque tournante Coriolis, où les expériences en fluides homogènes ou stratifiés sont réalisées dans une cuve de $14 \mathrm{~m}$ de diamètre et de $1 \mathrm{~m}$ de hauteur.

Toutes ces installations laissent présager pour la recherche grenobloise encore de riches moissons.

Je voudrais encore ajouter un mot à mes propos.

La préparation de cette conférence m'a permis de découvrir un travail dont j'avais entendu parler mais que je n'avais jamais eu l'occasion d'étudier en détail. Il s'agit du travail expérimental fait par Paul PERROUd comme mémoire de thèse de $\mathrm{Ph}$. D. à I'Université de Californie, à BERKEley en 1957.

Il porte sur l'étude expérimentale de la réflexion et de la diffraction des ondes solitaires. Ce travail de précurseur ne pouvait à l'époque n'être que partiellement éclairé par la théorie des ondes longues. Par contre, à la lumière des développements théoriques les plus récents, il prend un relief saisissant. Patience et longueur de temps...

\section{Bibliographie}

[1] GERTSNER F. (1887). - Théorie des vagues suivie d'un essai sur la théorie des profils de digues. Traduits et annotés par M. de Saint-Venant Ann. des Ponts et chaussées, pp. 31-86.

[2] AIRY G.B. (1845). - Tides and waves. Encyclopedia Metropolitana, pp. 241-396.

[3] Stokes G.G. (1849). - On the theory of oscillatory waves. Trans. Cambridge Phil. Sc. (8), pp. 441-455.

[4] HAvelock T.H. (1929). - Forced Surface waves on water. Phil Mag, pp. 569-576.

[5] DEAN W.R. (1945). - On the reflexion of surface waves by a submerged plane barrier. Prac. Cambridge Phil. Soc 41, pp. 231-238.

[6] MEİC \& LiU P.L.F. - Effects of Topography on the circulation in and near the surf zone in linear theory. Estuary Coastal Marine Sci. 5, pp. 25-37.

[7] BERKHOFF C.W. (1972). - Computation of combined refraction-diffraction. Proc 13th conf. Coastal Eng. ASCE 1 pp. 471-490.

[8] Russel J.S. (1945). - Report on waves. Rep. Meet. Brit. Ass. Adv. Sci. 14th York 1844. John Murray London, pp. 311-390. 